\title{
Erdbebengefährdungskarte für Österreich
}

\author{
Dipl. Ing., Stefan Weginger ${ }^{1}$; Dipl.-Ing. María del Puy Papí Isaba ${ }^{1}$; \\ Dipl.-Ing. Helmut Hausmann ${ }^{1}$; Dr. Wolfgang Lenhardt ${ }^{1}$ \\ ${ }^{1}$ Zentralanstalt für Meteorologie und Geodynamik, Wien
}

\section{Kurzfassung}

Gezeigt wird der aktuelle Stand der Neueinschätzung der Erdbebengefährdung für Österreich. Eine Aktualisierung der bisherigen Karte ist notwendig geworden, da die derzeit gültige Version bereits 25 Jahre alt ist und sich wesentliche Neuerungen ergeben haben. Die Verbesserungen der probabilistischen seismischen Gefährdungsbeurteilung (PSHA) beruhen auf einem erweiterten und aktualisierten Erdbebenkatalog, lokal ausgewählten und entwickelten Bodenbewegungsmodellen und der Anwendung neuer Berechnungsstandards. Der verwendete Ansatz kombiniert ein Modell seismischer Zonen, einen zonenfreien Ansatz und ein Modell mit geologischen Bruchzonen. Die Ergebnisse beinhalten die maximale horizontale Bodenbeschleunigung, die Antwortspektren sowie Gefährdungskurven. Sie sind für ganz Österreich für unterschiedliche Wiederkehrperioden und Bodenklassen online abrufbar um einen Vergleich mit der jetzigen Norm durchführen zu können.

\begin{abstract}
It is an international custom to review every $20-30$ years earthquake-resistant building codes, owing to technological, instrumental and scientific advances. The improvements of the new probabilistic seismic hazard assessment (PSHA) resulted from an expanded and updated earthquake catalogue, locally selected and developed ground motion models and the application of new calculation standards. The approach combines a model of seismic zones, a zone-free model and a model with geological fault zones. The results include the maximum horizontal ground acceleration, the response spectrum and hazard curves. They are available online for the area of Austria for different return periods and soil classes for reasons of comparison.
\end{abstract}

Vortrag Stefan Weginger

2. Wiener Dynamik Tage 2021 


\section{Ziel der Neueinschätzung}

Die Neueinschätzung der Erdbebengefährdung in Österreich erfolgte durch den Österreichischen Erdbebendienst der ZAMG. Ziel war die Bereitstellung der seismischen Lastenaufnahmen mittels der probabilistischen seismischen Gefährdungsbeurteilung (PSHA). Die Berechnung erfolgte für die maximale horizontale Bodenbeschleunigung (PGA) und für das horizontale Antwortspektrum (Uniform Hazard Spectrum - UHS) mit 5 \% Dämpfung. Die Ergebnisse sind für eine Überschreitungswahrscheinlichkeit von $2 \%, 5 \%$ und $10 \%$ innerhalb von 50 Jahren verfügbar. Das entspricht einer mittleren Wiederkehrperiode von 475, 975 und 2475 Jahren. Die entsprechenden horizontalen Belastungswerte wurden für einen Untergrund mit der Scherwellengeschwindigkeit $\left(V_{s 30}\right)$ von $800 \mathrm{~m} / \mathrm{s}$ berechnet und wurden für die Bodenklassen A, B, C und D bestimmt. Die Gefährdungskurven und UHS's der verschiedenen Bodenklassen und Wiederkehrperioden sind in einem interaktiven Internetportal zu Verfügung gestellt.

Eine Aktualisierung der bisherigen Gefährdungskarte [1] für Österreich ist notwendig geworden, da die derzeit gültige Version bereits mehr als 25 Jahre alt ist und sich wesentliche Neuerungen ergeben haben. Der Katalog wurde überarbeitet und mit der MomentenMagnitude erweitert. Umrechnungsbeziehungen zwischen messtechnisch erfassten Beben und den Ergebnissen der historischen Erdbebendaten konnten auf Grundlage einer größeren und höher qualitativen Datenbasis bestimmt werden. Eine neue Generation von Prognosemodellen der Bodenbewegungen (Ground Motion Prediction Equation - GMPE) liegt vor und es wurde zum ersten Mal lokal angepasste Erschütterungs-Abnahmegesetze für alle Bodenbewegungsparameter und der lokalen Intensität bestimmt. Dies trägt zu einer realistischeren Abschätzung der Erdebengefährdung bei. Die angeführten Ergebnisse entsprechen weitgehend den auf der D-A-CH Tagung 2019 präsentierten Ergebnissen.

\section{Seismizität}

Die Seismizität in Österreich und deren Auswirkungen können im europäischen Kontext als moderat eingestuft werden. Seit 1900 wurden in Österreich über zweitausend Erdbeben verspürt. Davon verursachten mehr als 50 Erdbeben leichte Gebäudeschäden entsprechend dem Intensitätsgrad 6 (EMS-98 [2]) und neun Erdbeben führten bereits zu teilweisen Gebäudeschäden (Intensitätsgrad im Epizentrum 7 bzw. 8). Erdbeben mit der Intensität 8 finden in Österreich in Abständen von ca. 100 bis 200 Jahren statt, welche sich entlang von tektonischen Störungszonen konzentrieren.

Die Datenbasis für die probabilistische Gefährdungsbeurteilung bildet der Österreichische Erdbebenkatalog (Austrian Earthquake Catalogue - AEC) [3]. Der Katalog wird ständig mit 
rezenten Erdbeben und den Ergebnissen der historischen Erdbebenforschung erweitert [4]. Der Katalog wurde ab Beginn der digitalen Aufzeichnung 1999 um die Momenten-Magnitude $(\mathrm{M} w)$ erweitert und daraus ein neues Verhältnis zur Lokalen Magnitude $(\mathrm{M} L)$ [5] abgeleitet. Für historische Beben wurden ein Verhältnis zur Intensität $\left(I_{0}\right)$ [6] bestimmt. Der AEC wurde weiteres mit den Daten der benachbarten Bebendienste aus dem Katalog des International Seismological Centres (ISC) verglichen und im $1^{\circ}$-Umkreis Österreichs ergänzt. Die Zeit der Katalogvollständigkeit wurde nach der "Slope-Methode“ [7] bestimmt. Zur genaueren Bestimmung der Tiefenverteilung der Hypozentren wurden jene Erdbeben selektiert, die definierte Lokalisierungskriterien erfüllen. Zusätzlich wurden bekannte Herdmechanismen integriert.

\section{Erdbebenmodell}

Neben der räumlichen Verteilung der Beben (Abbildung 3-1) wird die jährliche Wiederkehrhäufigkeit bestimmt. Die Magnitudenhäufigkeitsverteilung (Moment Frequency Distribution - MFD) zeigt die beobachtete jährliche Häufigkeit innerhalb von Magnitudenklassen und wird mit der „double-truncated Gutenberg-Richter” (GR) Verteilung durch die Parametern $a, b, M_{\min }$ und $M_{\max }$ beschrieben [8]. Sie beschreibt die kumulative jährliche Häufigkeitsrate $v$ (GI. 1) aller Beben im Magnitudenintervall zwischen $\mathrm{M}_{\text {Min }}$ und der maximale Magnitude $\mathrm{M}_{\mathrm{Max}}$.

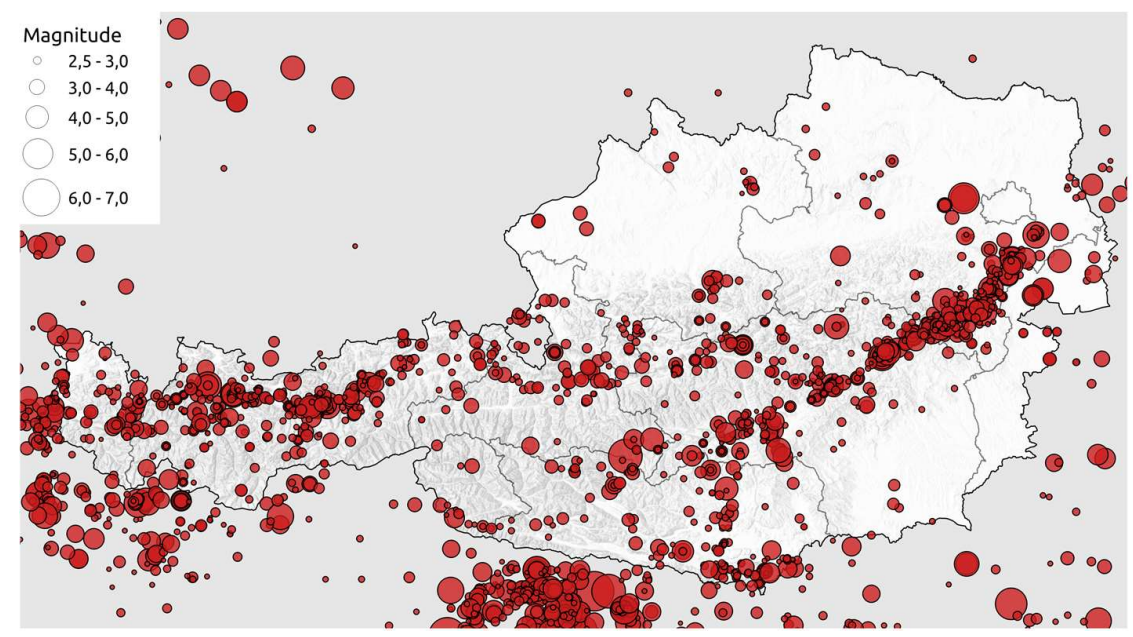

Abbildung 3-1: Seismizität in Österreich von 1000 bis 2018 [3]

$$
v\left(M_{\min }\right)=\int_{M_{\min }}^{M_{\max }} 10^{a-b m} d m
$$

Vortrag Stefan Weginger

2. Wiener Dynamik Tage 2021 
Abbildung 3-2 zeigt die inkrementelle und kumulierte MFD für das gesamte Untersuchungsgebiet (Österreich + einem $1^{\circ}$-Randbereich). Nach Weichert [8] wurde aus dem Datensatz ein regionaler b-Wert von 0,98 bestimmt, welcher auch gut mit jenen der Schweiz und Deutschland übereinstimmt [12].

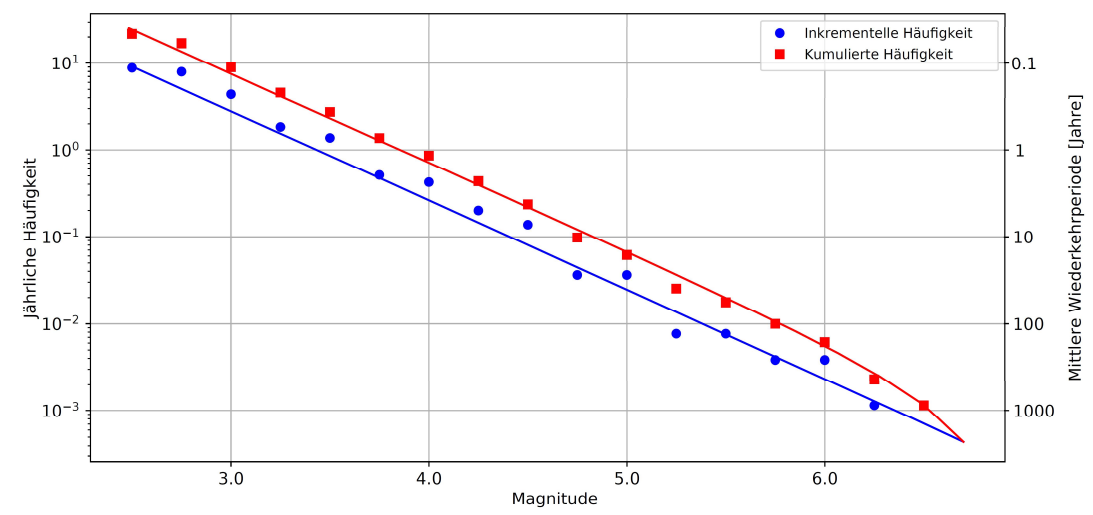

Abbildung 3-2: Inkrementelle und kumulierte MFD für Österreich $+1^{\circ}$

Da sich die MFD theoretisch bis ins Unendliche fortsetzt, wird sie von einer maximalen Magnitude begrenzt. Im Alpenraum können hinsichtlich der erforderlichen Bruchlänge Erdbeben der Magnitude > 8,0 ausgeschlossen werden. Erdbeben der Magnitude 6,0 - 6,5 sind jedoch aufgrund der allgemein kritisch gestressten Kruste überall möglich, auch wenn sie in Bereichen mit geringer Belastung sehr selten sind [9].

Die Schwierigkeit bei der Abschätzung von $\mathrm{M}_{\mathrm{Max}}$ in Gebieten mit moderater Seismizität ist die limitierte Beobachtungzeit. In vergleichbaren kontinentalen Regionen wurde der EPRI Ansatz [10][11] entwickelt und dieser wurde in den Nachbarländern angewandt [7][9][11]. Er basiert auf der Annahme, dass der Typ der Erdkruste (s.u.) ein Indikator für $\mathrm{M}_{\max }$ ist. Er ersetzt die zeitliche Limitierung räumlich in Form globaler Verteilungsfunktionen des entsprechenden Krustentyps. Nach den Kriterien der EPRI Studie ist der überwiegende Teil Österreichs (alpidische Kruste) der Zone „Extended Continental Crust“ zuzuordnen. Die nördlichen Gebiete (Bayern, Böhmische Masse) zählen zum Gebiet „Non-Extended Continental Crust“ [9][11][12][13]. Für Österreich ergibt sich nach EPRI ein $M_{\max } z$ wischen 6.0 und 6.5.

Zur Modellierung wird zwischen drei unterschiedlichen Ansätzen unterschieden:

1) Die großräumige Modellierung unterscheidet seismische Flächenquellen, die die räumliche Verteilung der Seismizität abbilden. Die übergeordneten Blöcke spiegeln den tektonischen Strukturaufbau wieder.

Vortrag Stefan Weginger

2. Wiener Dynamik Tage 2021 
2) Der zweite Ansatz ist ein zonenfreies Modell, indem mit unterschiedlichen KernelFunktionen die Seismizität geglättet wird.

3) Das dritte Modell gibt die seismisch aktiven tektonischen Bruchzonen wieder. Die Seismizität wird entlang der bekannten Störungszonen verteilt, da bei Wiederkehrperioden, die größer als der Beobachtungszeitraum sind, noch nicht alle Beben entlang einer Bruchzone beobachtet werden konnten.

Die MFD wird durch die Standardmethode nach Weichert bestimmt. Sind in Zonen mit geringer Seismizität nicht genügend Daten vorhanden um eine vertrauenswürdige Statistik zu rechnen, werden die Daten des übergeordneten Blockes berücksichtigt. Bei der Berechnung wurde das Konzept der Zonen und Superzonen [7] und der „Bayesian Penalized Maximum Likelihood“ Ansatz [9][13] verwendet.

Die Kombination aller Modelle und Parameter wird mit einem Entscheidungsbaum (LogicTree) durchgeführt. Die Berechnung ist in „Branching“-Levels unterteilt: a) seismische Modellierung, b) Bestimmung der MFD, c) Bestimmung von $\mathrm{M}_{\max }$ und d) Anwendung der GMPE. Zur Verfolgung und Bestimmung der Unsicherheiten wird jeder dieser Äste bei der Berechnung durchlaufen (Abbildung 3-3).

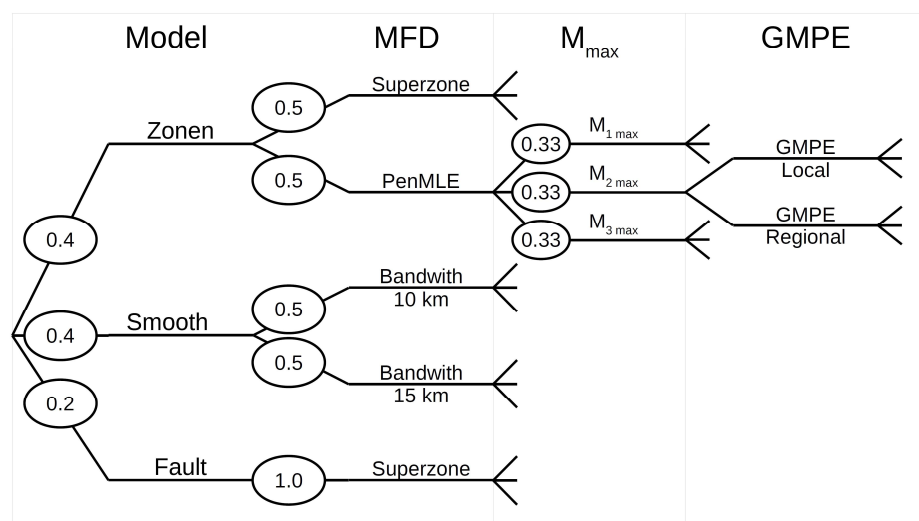

Abbildung 3-3: Entscheidungsbaum mit Wichtungswerten

„De-aggregation Plots“ tragen zum Verständnis der Bestimmung von Gefährdungunsicherheiten bei. Sie können für jeden Punkt und jeden Parameter berechnet werden. Die Höhe der Säulen (Abbildung 3-4) zeigt den Beitrag jeder Magnitude/Distanz Kombination bei der Gefährdungsberechnung. Am Beispiel für Wien wird hier deutlich, dass bei der 475-jährlichen Karte die Gefährdung hauptsächlich von Beben der Magnitude $M_{w} 5.0$ bis 5.5 aus einer Distanz von 35 - $45 \mathrm{~km}$ ausgeht.

Vortrag Stefan Weginger

2. Wiener Dynamik Tage 2021 


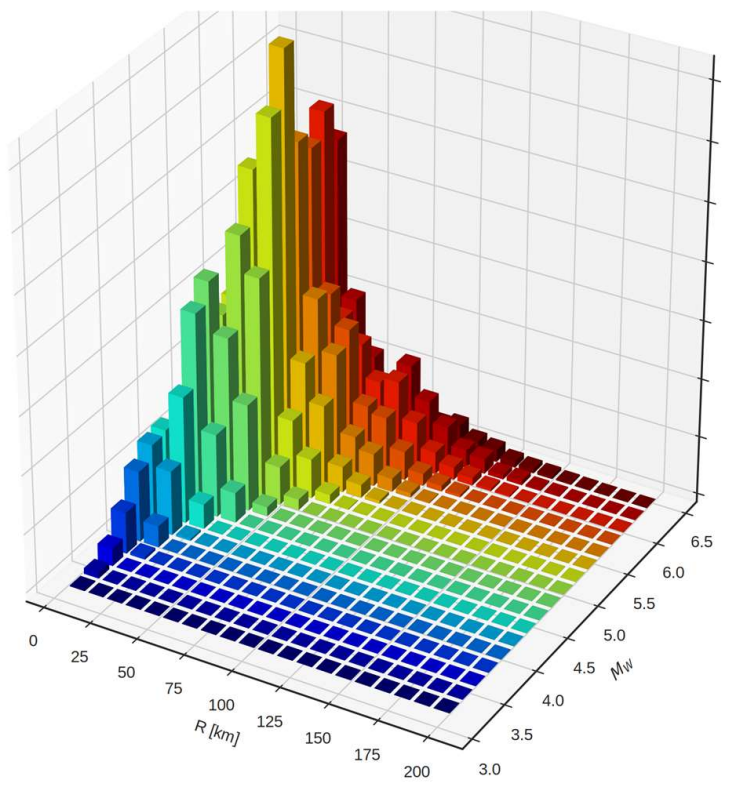

Abbildung 3-4: „De-aggregation plot“ für Wien (PGA, Wiederkehrperiode 475 Jahre). Die Höhe der Säulen zeigt den Beitrag jeder Magnitude/Distanz-Kombination zur Gefährdungsanalyse.

\section{Ergebnis}

Abbildung 4-5 zeigt die Erdbebengefährdungskarte (Stand April 2019) für PGA mit einer 10 prozentigen Überschreitungswahrscheinlichkeit in 50 Jahren entsprechend einer Wiederkehrperiode (Return Period, „RP“) von 475 Jahren. Die Karte gilt für Festgestein (Bodenklasse A nach EUROCODE 8). Der Vergleich mit dem Gefährdungsmodell in Abbildung 4-6 von Deutschland D2016 [9] zeigt eine gute Übereinstimmung. 


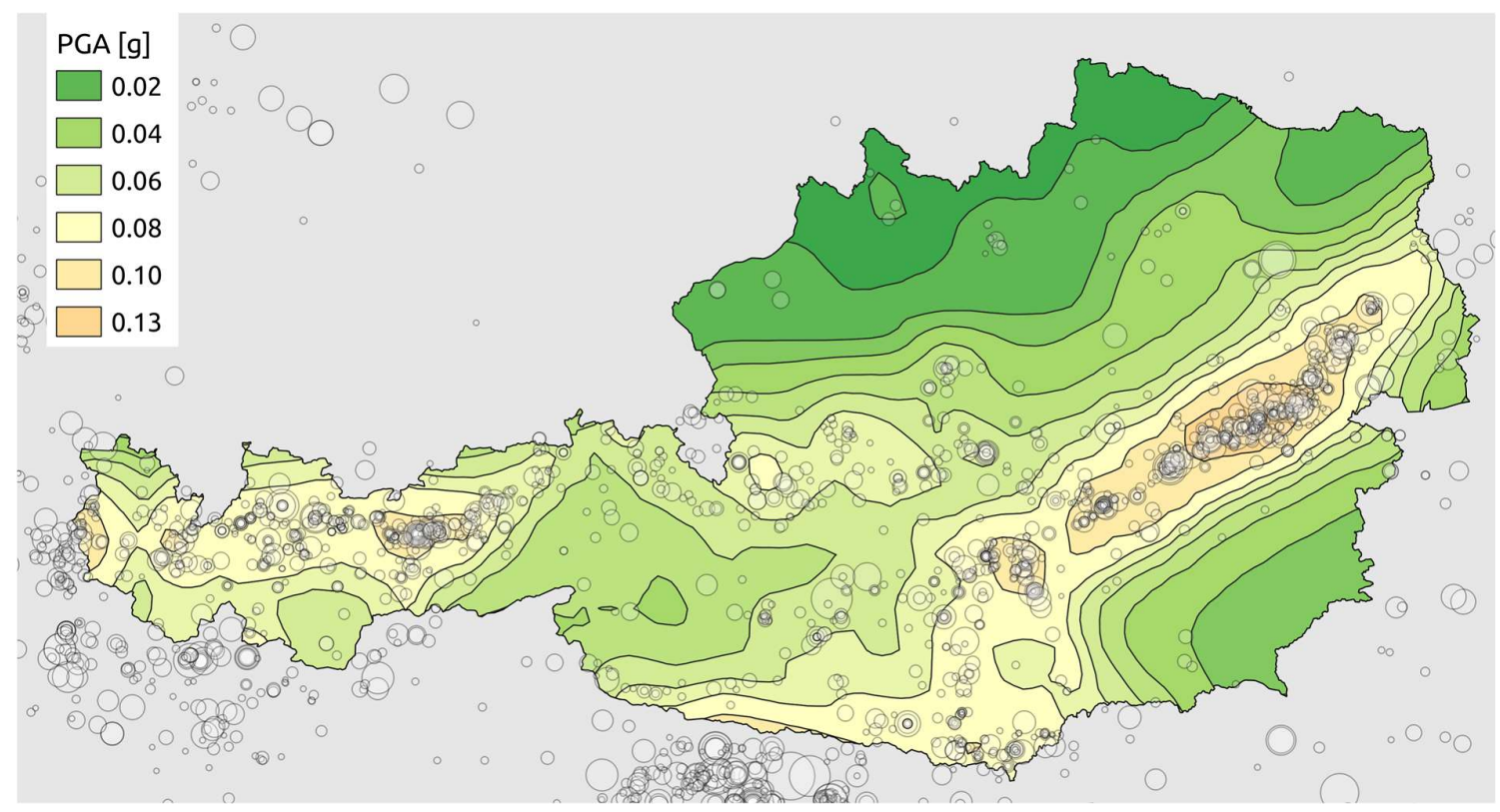

Abbildung 4-5: Erdbebengefährdungskarte (Stand April 2019) für PGA mit einer RP von 475 Jahren

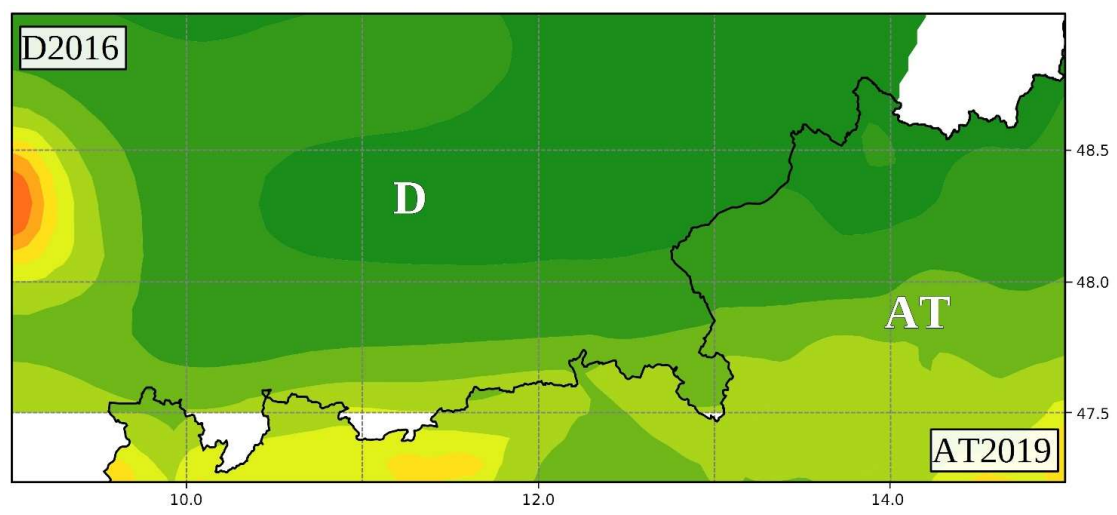

Abbildung 4-6: Vergleich mit dem Gefährdungsmodell von Deutschland (PGA, RP = 475 Jahre, $\left.\mathrm{Vs}_{30}=800 \mathrm{~m} / \mathrm{s}\right)$

Die Gefährdungskurven und UHS für jeden Rasterpunkt werden in einem interaktiven Internetportal (siehe Abbildung 4-7, http://geoweb.zamg.ac.at/hazard/dach19.html) zu Verfügung gestellt. Auswählbar sind die Wiederkehrperiode 475, 975 und 2475 Jahren und die Bodenklassen A, B, C und D. 
In diesem Zusammenhang erscheint es wichtig zu erwähnen, dass die neue Karte sich noch a) in einer Ergänzungsphase befindet, da ein Projekt, welches sich mit der Historischen Erdbebenerforschung in Tirol befasst, erst vor seinem Abschluss steht, und b) die bautechnischen Kriterien (inkl. möglicher Zonendefinitionen und Beaufschlagungswerte für verschiedene Bauwerksnutzungen, Bodenklassen), seitens der ÖNORM noch festgelegt werden müssen. Die verbesserte Gefährdungskarte wird dann als physikalische Basis für die neue Erdbeben-Kennwertekarte für Österreich dienen. Die Parameter hier, die in der neuen Norm herangezogen werden (Antwortspektrenwert bei $1 \mathrm{~Hz}$, ein Mittelwert von 1-10 Hz, etc.) sind derzeit in Diskussion. Jedenfalls gilt die bestehende ÖNORM 1998-1 bis die neue Regelung von der ÖNORM beschlossen und publiziert worden ist.

Erdbebenbemessungswert laut Gefährdungsstudie

Weginger, S. et al., 2019. Entwicklung einer regionalen Erdbebengefährdungskarte für Ōsterreich. D-A-C-H Tagungsband, Volume 16. Zentralanstalt für Meteorologie und Geodynamik
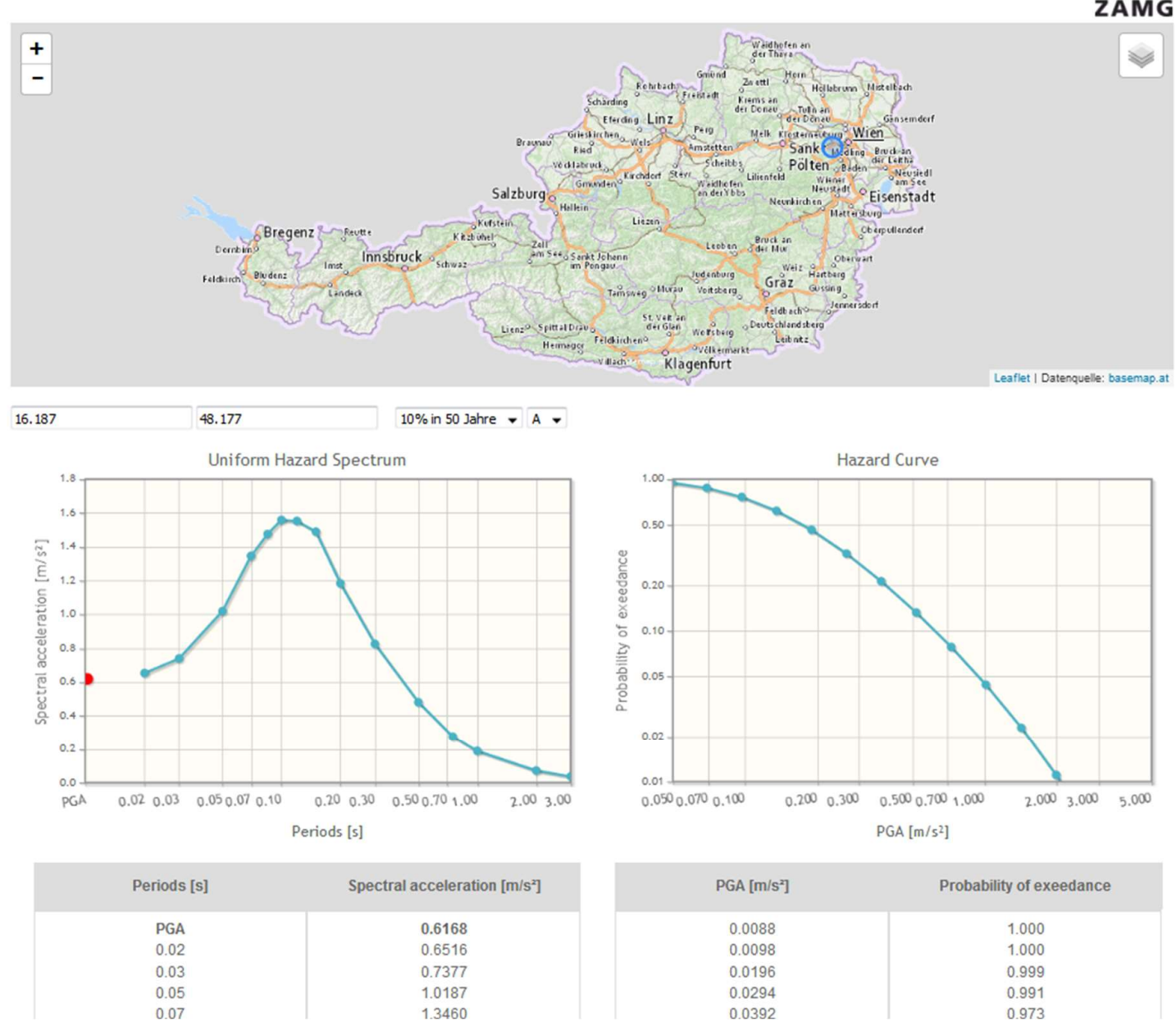

\begin{tabular}{|c|c|}
\hline PGA $\left[\mathrm{m} / \mathrm{s}^{2}\right]$ & Probability of exeedance \\
\hline 0.0088 & 1.000 \\
0.0098 & 1.000 \\
0.0196 & 0.999 \\
0.0294 & 0.991 \\
0.0392 & 0.973 \\
\hline
\end{tabular}

Abbildung 4-7: Abfragetool für Kennwerte der Erdbebengefährdung

Vortrag Stefan Weginger

2. Wiener Dynamik Tage 2021 


\section{Zusammenfassung}

Für die Berechnung der seismischen Gefährdung Österreichs wurden international erprobte und übliche Methoden angewendet. Die neue Gefährdungskarte beruht einerseits auf einem erweiterten Datenbestand rezenter Erdbeben und andererseits neuen Ergebnissen der historischen Erdbebenforschung. Die verbesserte Messnetzdichte erlaubt eine bessere Bestimmung der Herdtiefen und Bebenmechanismen. Durch die Einhaltung internationaler Vorgaben und Berechnungsmethoden wird das Ergebnis auch vergleichbar in den Grenzbereichen, wie eine Gegenüberstellung mit dem deutschen Gefährdungsmodell D2016 und der Schweizer Gefährdungskarte zeigte. Solange die ÖNORM keine Aktualisierung publiziert, gilt die bestehende Baunorm.

Literaturangaben:

[1] Lenhardt, W. (1995). Regional earthquake hazard in Austria. In: $10^{\text {th }}$ European Conference on Earthquake Engineering.

[2] Grünthal, G. (ed.) (1998). European Macroseismic Scale 1998 (EMS-98). Conseil de l'Europe, Cahiers du Centre Européen de Géodynamique et de Seismologie, Luxembourg.

[3] AEC, 2018. Austrian Earthquake Catalogue from 1000 to 2018 A.D., Zentralanstalt für Meteorologie und Geodynamik, Abteilung Geophysik, 1190 Wien.

[4] Hammerl, C., \& Lenhardt, W. (2013). Erdbeben in Niederösterreich von 1000 bis 2009 n. Chr. Abhandlung der Geologischen Bundesanstalt.

[5] Weginger, S., Jia, Y., Papí-Isaba, M. \& Lenhardt, W. (2018). Towards a new seismic hazard map of Austria. In: European Seismological Commission General Assembly. Malta.

[6] Papí-Isaba, M., Jia, Y. \& Weginger, S. (2018). Verification of the intensity prediction equation for Austria. In European Seismological Commission General Assembly. Malta.

[7] Grünthal, G., Stromeyer, D., Bosse, C., Cotton, F., \& Bindi, D. (2018). The probabilistic seismic hazard assessment of Germany-version 2016. Bulletin of Earthquake Engineering, 16 (10).

[8] Weichert, D. (1980). Estimation of the earthquake recurrence parameter for unequal observations periods for different magnitudes. Bulletin of the Seismological Society of America, 70 (4), 1337-1356.

[9] Wiemer, S., Danciu, L., Edwards, B., Marti, M., Fäh, D. Hiemer, S., Woessner, J., Cauzzi, C., Kästli, P. \& Kremer, K. (2015). Seismic Hazard Model 2015 for Switzerland (SUlhaz2015). Swiss Seismological Service (SED) at ETH Zurich.

Vortrag Stefan Weginger

2. Wiener Dynamik Tage 2021 
[10] Stirewalt, L., McDuffie, S., Coppersmith, K. \& Fuller, C. (2012). Central and Eastern United States Seismic Source Characterization for Nuclear Facilities. U.S. Nuclear Regulatory Commission.

[11] Burkhard, M. \& Grünthal, G. (2009). Seismic source zone characterization for the seismic hazard assessment project PEGASOS by the Expert Group 2 (EG1b). Swiss Journal of Geosciences, 102(1), 149-188.

[12] Grünthal, G., Bosse, C. \& Stromeyer, D. (2009). Die neue Generation der probabilistischen seismischen Gefährdungseinschätzung der Bundesrepublik Deutschland: Version 2007. Deutsches GeoForschungsZentrum GFZ.

[13] Woessner, J., Laurentiu, D., Giardini, D., Crowley, H., Cotton, F. \& Grünthal, G. (2015). The 2013 European Hazard Model: key components and results. Bulletin Earthquake Engineering, 13(12), 3553-3596.

Autorenkontakt: s.weginger@zamg.ac.at 